\title{
Spontaneous and Evoked Release Are Independently Regulated at Individual Active Zones
}

\author{
Jan E. Melom, Yulia Akbergenova, Jeffrey P. Gavornik, and J. Troy Littleton \\ The Picower Institute for Learning and Memory, Department of Biology and Department of Brain and Cognitive Sciences, Massachusetts Institute of \\ Technology, Cambridge, Massachusetts 02139
}

\begin{abstract}
Neurotransmitter release from synaptic vesicle fusion is the fundamental mechanism for neuronal communication at synapses. Evoked release following an action potential has been well characterized for its function in activating the postsynaptic cell, but the significance of spontaneous release is less clear. Using transgenic tools to image single synaptic vesicle fusion events at individual release sites (active zones) in Drosophila, we characterized the spatial and temporal dynamics of exocytotic events that occur spontaneously or in response to an action potential. We also analyzed the relationship between these two modes of fusion at single release sites. A majority of active zones participate in both modes of fusion, although release probability is not correlated between the two modes of release and is highly variable across the population. A subset of active zones is specifically dedicated to spontaneous release, indicating a population of postsynaptic receptors is uniquely activated by this mode of vesicle fusion. Imaging synaptic transmission at individual release sites also revealed general rules for spontaneous and evoked release, and indicate that active zones with similar release probability can cluster spatially within individual synaptic boutons. These findings suggest neuronal connections contain two information channels that can be spatially segregated and independently regulated to transmit evoked or spontaneous fusion signals.
\end{abstract}

\section{Introduction}

Neurotransmitters can be released during evoked fusion following an action potential, or through spontaneous fusion of vesicles (termed "minis") in the absence of nerve stimulation (Fatt and Katz, 1952; Katz and Miledi, 1969). The core fusion machinery required for both spontaneous and evoked release is the SNARE complex, a family of membrane-tethered proteins that assemble into an $\alpha$-helical coiled-coil structure that bridges the synaptic vesicle and plasma membrane (Söllner et al., 1993). The two modes of vesicle release have been found at most synapses and are assumed to occur across the same population of active zones. However, genetic manipulations of several SNARE-binding proteins have revealed that evoked and spontaneous release can be differentially regulated (Littleton et al., 1993; Yoshihara et al., 1999; Huntwork and Littleton, 2007; Maximov et al., 2007; Pang et al., 2011). Indeed, spontaneous and evoked release may share separate SNARE fusion proteins (Hua et al., 2011; Ramirez et al., 2012), or represent release from distinct vesicle pools (Fredj and Burrone, 2009; Sara et al., 2011; Ramirez et al., 2012). Activitydependent plastic changes in presynaptic release can also differentially regulate spontaneous versus evoked fusion (Yoshihara et

Received Aug. 5, 2013; revised Sept. 5, 2013; accepted Sept. 12, 2013.

Author contributions: J.E.M., Y.A., J.P.G., and J.T.L. designed research; J.E.M., Y.A., and J.P.G. performed research; J.E.M., Y.A., J.P.G., and J.T.L. analyzed data; J.E.M., Y.A., J.P.G., and J.T.L. wrote the paper.

This work was supported by NIH Grant NS40296 to J.T.L. We thank Pamela Russell for help with statistical analysis and Loren Looger for GCaMP CDNAs.

The authors declare no competing financial interests.

Correspondence should be addressed to J. Troy Littleton at the above address. E-mail: troy@mit.edu.

DOI:10.1523/JNEUROSCI.3334-13.2013

Copyright $\odot 2013$ the authors $\quad 0270-6474 / 13 / 3317253-11 \$ 15.00 / 0$ al., 2005; Nosyreva et al., 2013), depending upon the synapse or stimulation paradigm.

Whether receptors on the postsynaptic cell have the ability to distinguish between neurotransmitters released through the two independent fusion pathways is debated (Sara et al., 2005; Groemer and Klingauf, 2007; Atasoy et al., 2008). This is a critical point for characterizing information flow at synapses. If both vesicle release mechanisms activate the same set of receptors, crosstalk would occur between the two modes of fusion. However, if spontaneous release occurs at distinct release sites, the postsynaptic cell may be capable of differentiating between the two modes of release, suggesting spontaneous fusion may represent a separate information channel independent of the traditional $\mathrm{Ca}^{2+}$-activated evoked release pathway. The major confound to this question has been the inability to examine vesicle fusion at individual active zones. Classical electrophysiological studies of synaptic transmission measure the postsynaptic effect of neurotransmitter release over a large population of release sites, precluding an analysis of how individual active zones participate in and regulate these two modes of vesicle fusion. Here, we have used $\mathrm{Ca}^{2+}$ imaging of postsynaptic glutamate receptor activation following vesicle fusion to visualize all exocytotic events occurring through both spontaneous and evoked release pathways at Drosophila glutamatergic neuromuscular junctions (NMJs), allowing us to define general rules for vesicle fusion events at single active zones.

\section{Materials and Methods}

Drosophila genetics and molecular biology. Flies were cultured on standard medium at $25^{\circ} \mathrm{C}$. Flies of both sexes were used for all experiments. mef2-Gal4 was used to drive transgene expression in muscle. The fluorescent $\mathrm{Ca}^{2+}$ sensor GCaMP5C was tethered to the plasma membrane 
with an N-terminal myristoylation (myr) sequence. The first 90 aa of Src64b, containing a myristoylation target sequence, were subcloned into pBI-UASc with EcoRI and BglII (creating pBI-UASc-myr). GCaMP5G (Addgene plasmid 31788) cDNA was cloned into pBI-UASc-myr with NotI and XbaI. UAS-myrGCaMP5 was injected using $\phi$-C-31 transformation (Genetic Services).

Spontaneous release $\mathrm{Ca}^{2+}$ imaging. Wandering third instar larvae expressing postsynaptic UAS-myrGCaMP5 were dissected in $\mathrm{Ca}^{2+}$-free HL3.1 saline (in mM: $70 \mathrm{NaCl}, 5 \mathrm{KCl}, 4 \mathrm{MgCl}_{2}, 0.2 \mathrm{CaCl}_{2}, 10 \mathrm{NaHCO}_{3}, 5$ Trehalose, 115 sucrose, 5 HEPES-NaOH, pH 7.2) at room temperature. The motor nerves were carefully snipped below the ventral nerve cord, and the CNS was removed. The preparation was washed several times with HL3.1 containing $1.5 \mathrm{mM} \mathrm{Ca}^{2+}$ and allowed to rest for $5 \mathrm{~min}$. We acquired images with a PerkinElmer Ultraview Vox spinning disc confocal microscope equipped with a Hamamatsu C9100-13 ImagEM EM $\mathrm{CCD}$ at $8-35 \mathrm{~Hz}$ with a $40 \times 0.8 \mathrm{NA}$ water-immersion objective (Carl Zeiss). A single confocal plane of muscle $4 \mathrm{NMJ}$ in segments A3 or A4 was continuously imaged for 3-10 $\mathrm{min}$ (average duration was $4.9 \mathrm{~min}$ ). Slight $z$-drift was manually corrected during the imaging session. Imaging sessions in which significant movement of the muscle occurred were discarded.

Concurrent evoked and spontaneous release $\mathrm{Ca}^{2+}$ imaging. Wandering third instar larvae were dissected in $\mathrm{Ca}^{2+}$-free HL3 containing $20 \mathrm{~mm}$ $\mathrm{MgCl}_{2}$. After dissection, preparations were maintained in HL3 with 20 $\mathrm{mM} \mathrm{MgCl}$ and $1 \mathrm{mM} \mathrm{Ca}^{2+}$ for $5 \mathrm{~min}$. We found that increased $\mathrm{Mg}^{2+}$ and slightly decreased $\mathrm{Ca}^{2+}$ inhibited muscle contraction without significant dimming of $\mathrm{Ca}^{2+}$-bound myrGCaMP5 fluorescence. To stimulate the $\mathrm{NMJ}$, motor nerves cut close to the ventral ganglion were sucked into a pipette. Single pulses of current were delivered every $3 \mathrm{~s}$ with the A.M.P.I. Master- 8 stimulator using a stimulus strength just above the threshold for evoking EJPs. A single focal plane was imaged continuously for 4-10 min.

$\mathrm{Ca}^{2+}$ imaging ROI acquisition. We used Volocity 3D Image Analysis software (PerkinElmer) to analyze images. All images were Gaussian filtered (fine) to reduce noise and a movement-correction algorithm was applied. To enhance identification of myrGCaMP5 flashes, background myrGCaMP fluorescence was subtracted by creating a composite stack of 5-6 images during which no synaptic release occurred. To analyze spontaneous or evoked activity, regions of interest (ROIs) were manually drawn within the center of peak fluorescence $\left(>50 \% \Delta F / F_{\text {avg }}\right)$ with an average area of 0.76 $\mu \mathrm{m}^{2}$. ROIs were deemed separate if their spatial boundaries did not overlap by $>30 \%$. Over several minutes of imaging, the fluorescence spread of $\mathrm{Ca}^{2+}$ events assigned to the same ROI was consistent within $1-2$ pixels $(0.219$ $\mu \mathrm{m} /$ pixel). For analysis of fluorescence changes we used the original file without background subtraction. The fluorescence of dark areas removed from the NMJ were used for background fluorescence and subtracted. $\Delta F$ was estimated by subtraction of baseline fluorescence of each ROI from mean fluorescence intensity at the peak of each flash. The time and location of $\mathrm{Ca}^{2+}$ events were imported into Matlab for further analysis. All errors are reported as SEM unless noted.

$\mathrm{Ca}^{2+}$ imaging ROI analysis. ROI location (coordinates and size), $\mathrm{Ca}^{2+}$ event times, and fluorescence levels were imported into MATLAB (MathWorks) for subsequent analysis. Spontaneous event distributions were estimated within each experiment by plotting the number of ROIs with each observed event count as a percentage of the total number of ROIs identified at the NMJ. To test whether the data were consistent with the hypothesis that spontaneous events occur independently with Poisson statistics, the maximum likelihood estimate (MLE) of the intensity parameter $\lambda$ was calculated for a Poisson distribution truncated to exclude the probability of zero events (necessary since release failures do not produce a measurable signal) and scaled by the total experiment time to units of events/min. Pearson's $\chi^{2}$ goodness of fit test was used to compare the number of observed events with the number of events expected assuming a Poisson distribution with the fit intensity parameter multiplied by the total experiment time. The cumulative distribution was estimated by pooling event counts across all experiments.

To quantify the observation that relatively active ROIs cluster together in space, "highly active" ROIs were defined as those whose spontaneous event rate was greater than two SDs from the mean event rate across all
ROIs within an experiment. The remaining ROIs (excluding the "highly active") were classified as being either "near" $(<2.5 \mu \mathrm{m})$ or "far" $(\geq 2.5$ $\mu \mathrm{m})$ from an active ROI and the event rate of each population was calculated as a percentage of the average event rate across both the near and far populations. A value of $120 \%$, for example, for the near population would mean that ROIs within $2.5 \mu \mathrm{m}$ of the highly active ROIs have a spontaneous activity rate $20 \%$ higher than the average rate of all non-highly active ROIs. These metrics were calculated for each experiment and compared using a paired $t$ test (normality confirmed via the Shapiro-Wilk test).

The evoked event probability distribution was generated by binning and counting the individual event probabilities for all ROIs (defined as the ratio of the evoked event count to stimulus count). These data were fit to a gamma distribution using MLE and the $\chi^{2}$ test was used to assess goodness of fit. To determine correlation between evoked and spontaneous activity levels, the spontaneous event rate was plotted as a function of the evoked rate for each ROI that contained both spontaneous and evoked activity. These data were fit with a linear regression and the residuals were used to calculate the coefficient of determination, $R^{2}$. The Pearson correlation coefficient, $\rho$, was also calculated to determine linear correlation within the data.

Immunohistochemistry. Larvae were fixed for 40 min in HL3.1 containing $4 \%$ formaldehyde. Following washes in PBS and PBST (1\% Triton $\mathrm{X}-100)$, larvae were incubated overnight with primary antibody at $4^{\circ} \mathrm{C}$, incubated with secondary antibodies for $4 \mathrm{~h}$ at room temperature the following day, and mounted in $70 \%$ glycerol for imaging. Antibodies were diluted as follows: mouse nc82 (anti-Brp, 1:100), Rhodamine redconjugated goat anti-mouse (1:1000), Cy3-conjugated mouse-antiGluRIIIC (1:100).

Electrophysiology. For simultaneous recording of postsynaptic currents during myrGCaMP5 imaging, sharp microelectrodes filled with $3 \mathrm{M} \mathrm{KCl}$ (30-50 $\mathrm{M} \Omega$ resistance) were placed into the muscle under a $40 \times$ objective. Recordings were performed using an Axopatch 200B (Molecular Devices) amplifier equipped with a CV203BU head stage. Data acquisition was performed using Axoscope 10 software (Molecular Devices). A mini analysis program (Synaptosoft) was used for spontaneous recording analysis. Only recordings with resting membrane potential below $-60 \mathrm{mV}$ and stable background noise were analyzed. Time points for each event were manually correlated with myrGCaMP5 signals observed during concurrent imaging.

\section{Results}

\section{Postsynaptic expression of myristoylated GCaMP5 reveals glutamate receptor responses to single synaptic vesicle fusion events}

The Drosophila NMJ shares basic molecular components of synaptic transmission with those found at most synapses and is ideally suited to address spatial properties of release at individual active zones. The NMJ contains spatially segregated active zones (termed T-bars) that oppose a distinct population of clustered glutamate receptors in the postsynaptic muscle (Fig. 1A). This spatial arrangement of release sites and clustered receptors has been used to analyze evoked release probability at individual release sites by measuring $\mathrm{Ca}^{2+}$ influx that occurs through postsynaptic glutamate receptors following vesicle fusion (Peled and Isacoff, 2011). Prior $\mathrm{Ca}^{2+}$ imaging techniques used at the NMJ could not identify all vesicle fusion events, limiting the analysis of evoked versus spontaneous release properties (Desai and Lnenicka, 2011; Peled and Isacoff, 2011). To expand upon the utility of this method to visualize exocytotic events, we generated a myristoylated (membrane-tethered) variant of a genetically encoded $\mathrm{Ca}^{2+}$-sensitive GFP (GCaMP5) that robustly detected postsynaptic responses to both evoked and spontaneous synaptic vesicle fusion events when visualized with a spinning disc confocal microscope. UAS-myrGCaMP5 was targeted to the inner leaflet of the postsynaptic plasma membrane when driven by the muscle driver mef2-GAL4. Drosophila motor axons exit the brain 
A
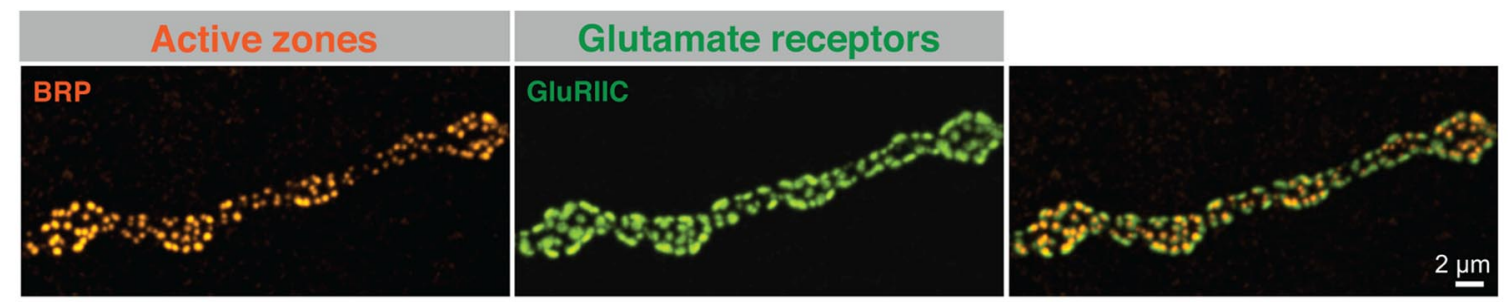

B

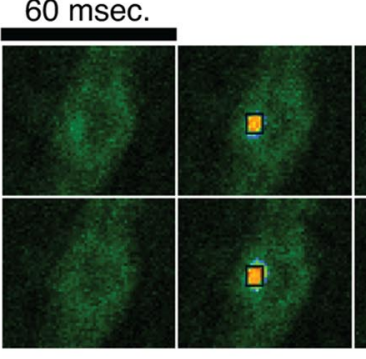

C
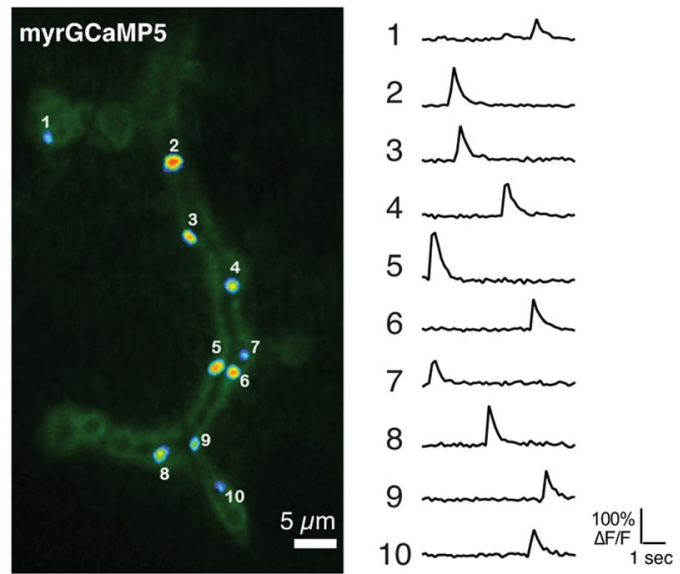

$\mathbf{E}$

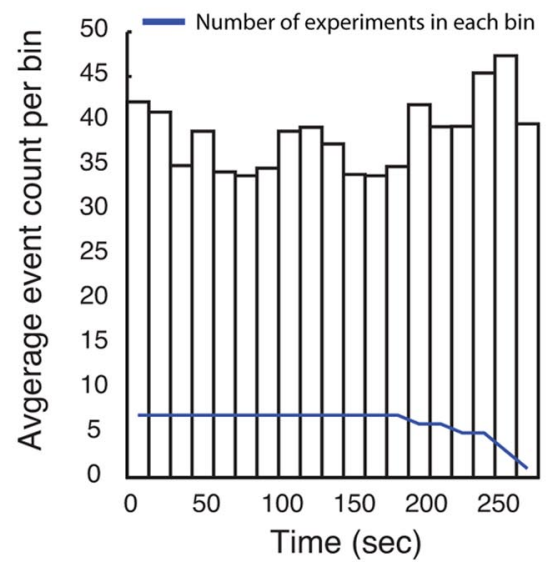

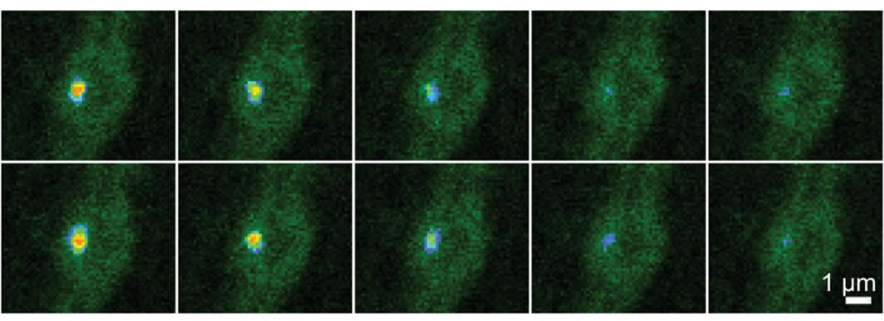

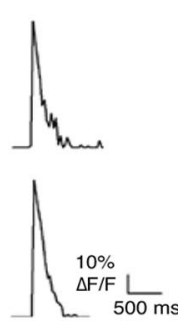

D
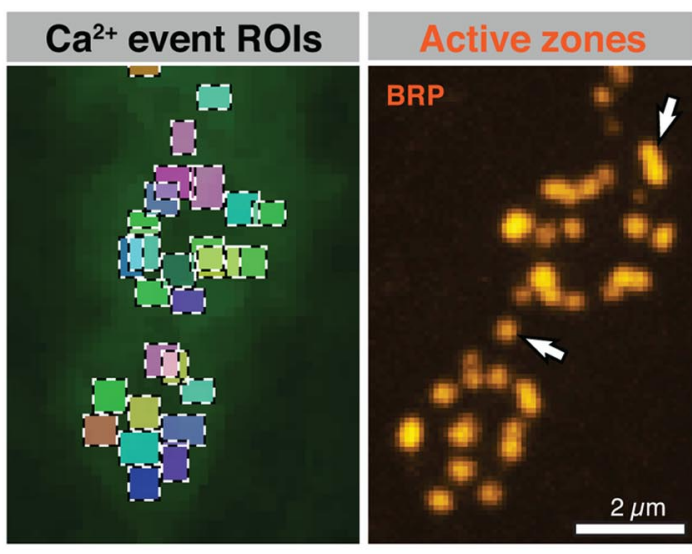

$\mathbf{F}$

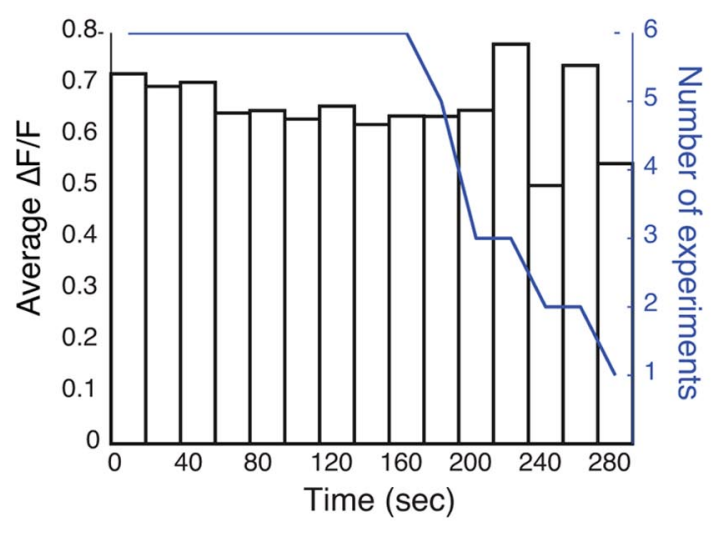

Figure 1. Spontaneous neurotransmission visualized at single active zones. $A$, Immunostaining at muscle fiber 4 for the active zone protein BRP (orange) and glutamate receptor subunit GluRIIC (green). Individual active zones are opposed by glutamate receptor clusters along en passant boutons. $\boldsymbol{B}$, Two series of consecutive images of a single bouton expressing postsynaptic myrGCaMP5 following separate spontaneous fusion events at the same R0I (boxed). Baseline myrGCaMP5 fluorescence (green) and the change in myrGCaMP5 fluorescence above background (rainbow) at a single ROl is shown on the left, with the corresponding $\Delta F / F$ traces on the right. C, Compressed imaging stack for a $10 \mathrm{~s}$ recording period for an NMJ expressing myrGCaMP5 postsynaptically. The fluorescence change in myrGCaMP5 above background for the 10 ROIs indicated is shown on the right. $\boldsymbol{D}$, Comparison of ROIs encompassing peak fluorescence $\mathrm{Ca}^{2+}$ flashes (multiple colors) recorded in live animals at two synaptic boutons versus postfixation staining for the active zone marker $\mathrm{BRP}$ (orange). $\boldsymbol{E}$, The average $\mathrm{Ca}^{2+}$ event rate per 20 s bin remains relatively constant over the duration of all experiments. $F$, The average $\Delta F / F$ per $20 \mathrm{~s}$ bin remain relatively constant over the duration of all experiments. In both plots, the blue line indicates the number of experiments used to estimate the average for each bin (decreases occur as shorter duration experiments end).

and innervate defined muscle fibers through an axonal terminal that arborizes to form branches and en passant synaptic boutons, each of which contain multiple individual active zones. Infoldings of the muscle plasma membrane surround synaptic boutons, forming a subsynaptic reticulum that effectively concentrates myrGCaMP5. We focused our analysis on the large type $1 \mathrm{~b}$ NMJs at larval muscle fiber 4 due to its relatively compact and simple arborization pattern. This synaptic connection contains sev- 
A
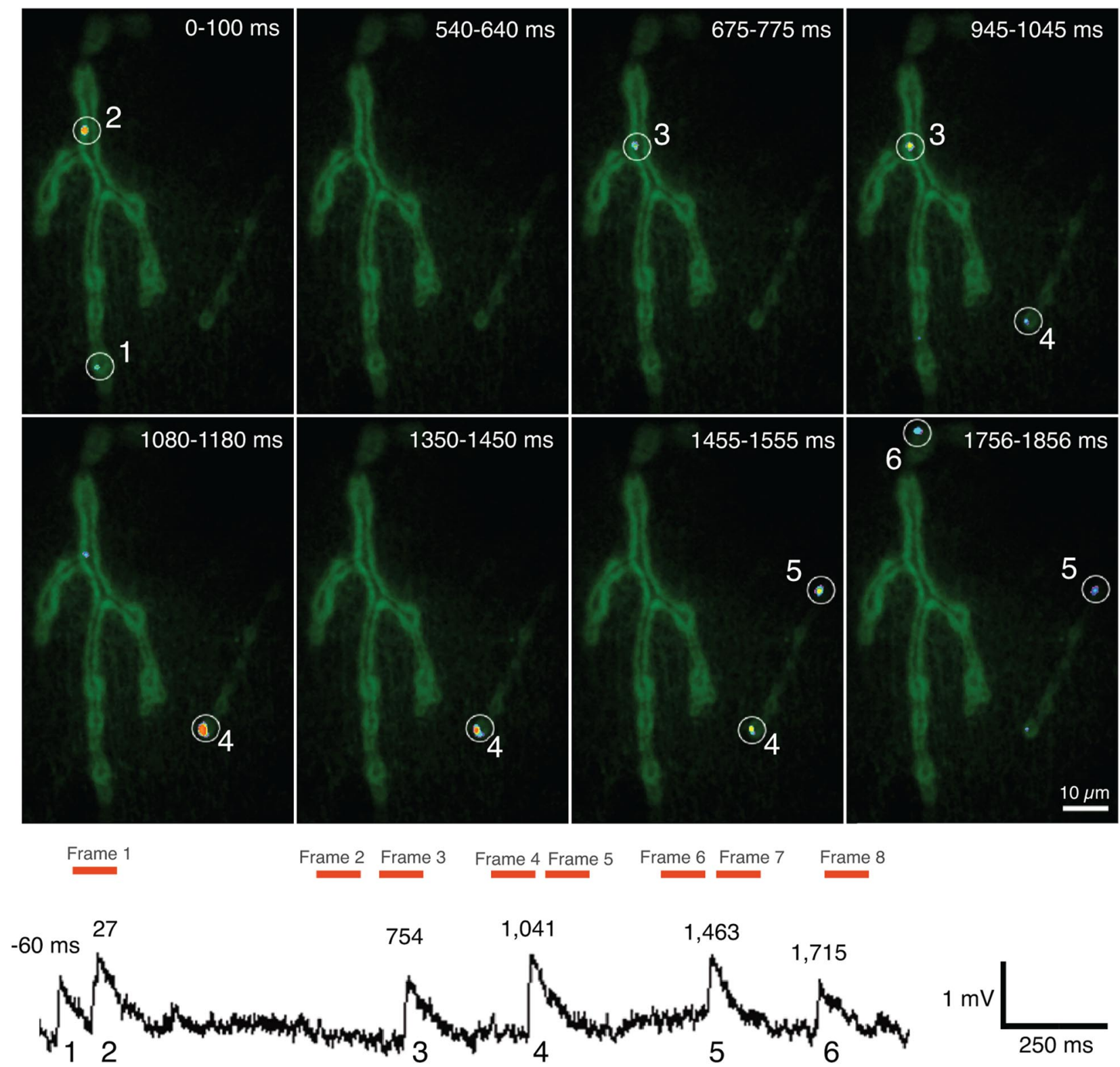

B
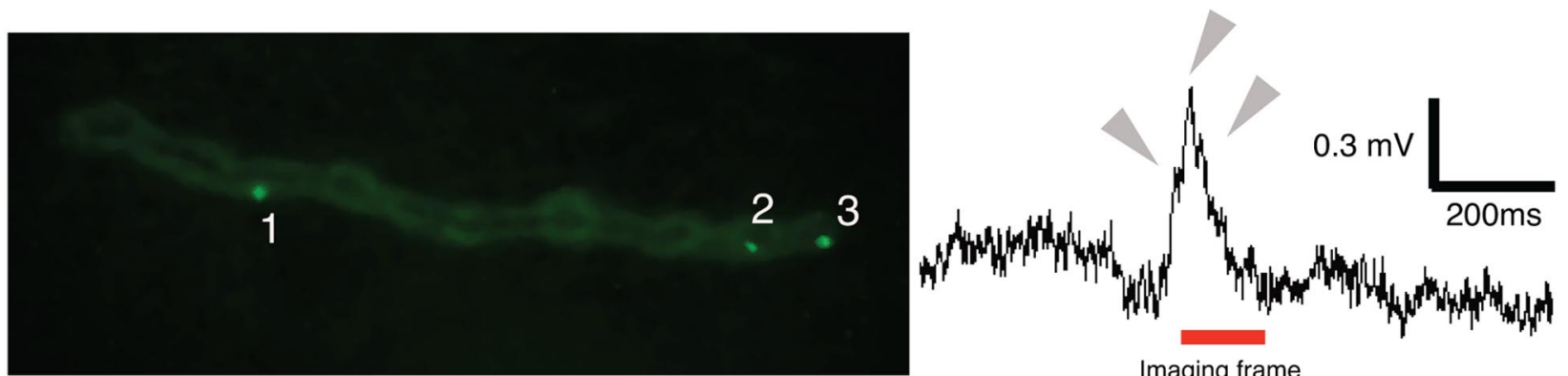

Figure 2. Correlation of spontaneous $\mathrm{Ca}^{2+}$ events with electrophysiological recordings. $\boldsymbol{A}$, Eight continuous imaging frames are shown in which $\mathrm{Ca}^{2+}$ events were detected at five ROIs in a muscle 4 NMJ. Simultaneous sharp electrode recordings from the muscle revealed spontaneous miniature EPSPs that correlated with the observed $\mathrm{Ca}^{2+}$ events. $\boldsymbol{B}$, In rare cases where extra $\mathrm{Ca}^{2+}$ events were observed during recordings, closer inspection of the waveforms revealed multiple peaks, suggesting near simultaneous fusion of multiple vesicles that were difficult to separate physiologically. Here, three spatially localized $\mathrm{Ca}^{2+}$ events map to a miniature EPSP event with three separate peaks.

eral hundred individual active zones that form contacts with the muscle (Fig. 1A). Each synaptic bouton contains between 5 and 30 active zones that are spatially separated and opposed by a cluster of glutamate receptors, providing a one-to-one match between the release machinery and the postsynaptic receptive unit (Fig. 1A).

Transgenic lines expressing postsynaptic myrGCaMP5 showed robust and spatially segregated signals for $\mathrm{Ca}^{2+}$ influx 
A
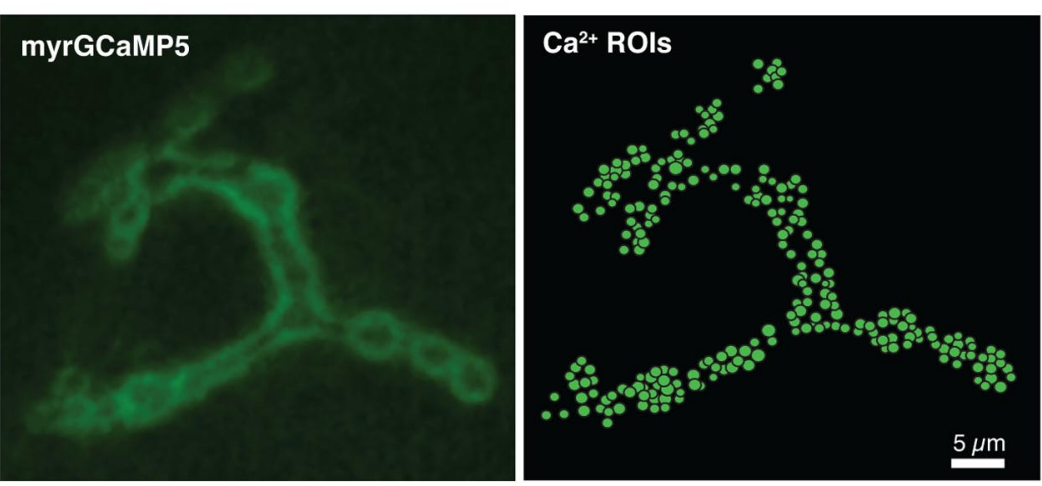

C

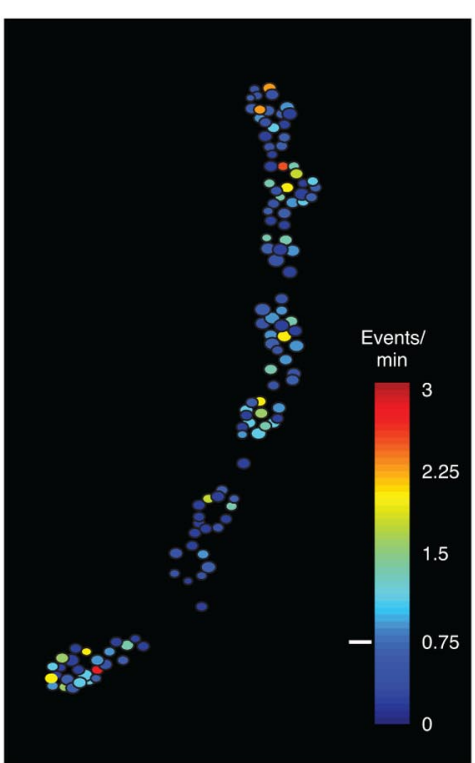

E

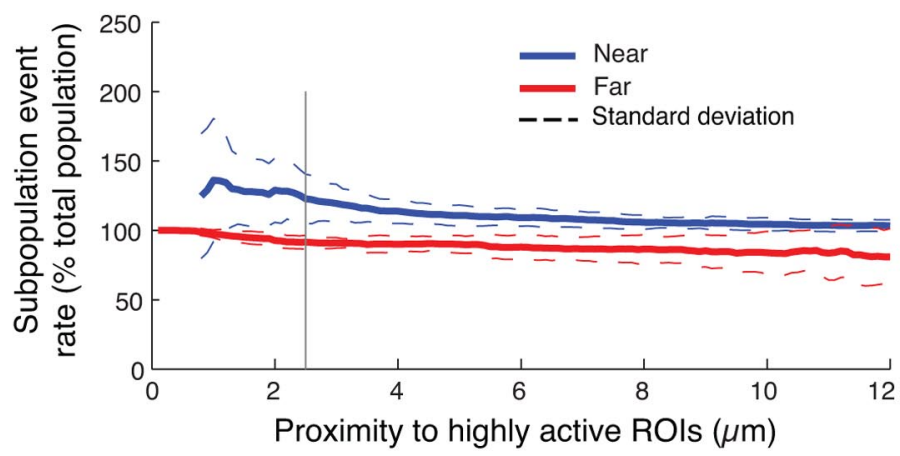

B

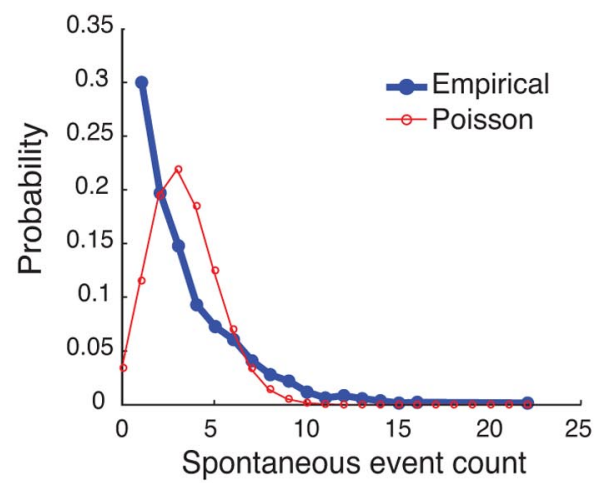

D

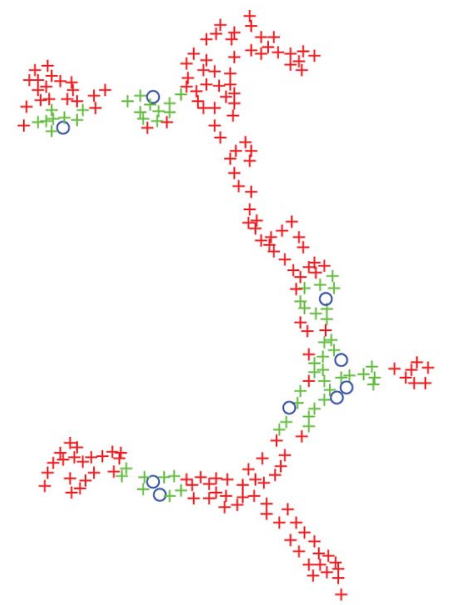

$5 \mu \mathrm{m}$

Figure 3. Properties of spontaneous neurotransmission. $A$, Baseline expression of myrGCaMP5 at an NMJ (left panel, green) compared with the sites of spontaneous $\mathrm{Ca}^{2+}$ events annotated with circular ROIs recorded over a 4 min imaging session. $\boldsymbol{B}$, The empirical spontaneous event count probability distribution (blue) does not match ( $p<0.001, \chi^{2}$ goodness of fit test) the best fit Poisson distribution (red, $\lambda=0.69$ event/min, see methods). C, Pseudo-color activity heatmaps showing the distribution of spontaneous synaptic activity across two example NMJs. Each circle represents a single ROI with the size coding for ROl area and color coding for event rate. White lines next to the colorbar mark the average event rate over all ROls. D, To quantify the observation that active ROls form spatial clusters, ROls were assigned to subpopulations indicating whether that they were near ( $<2.5 \mu \mathrm{m}$, green) or far ( $\geq 2.5 \mu \mathrm{m}$, red) from highly active ROls ( $>2$ SDs above average event rate, blue). $\boldsymbol{E}$, Plotting the average event rate of the two populations as a percentage of the mean rate across both populations reveals that ROIs near highly active ROIs have higher event rates than those far away. SDs (dashed lines) scale with respective population sizes, which are a function of the local radius (the vertical line marks $2.5 \mu \mathrm{m}$ ). $\boldsymbol{F}$, There is a significant difference in population event rates within a $2.5 \mu \mathrm{m}$ local radius $\left({ }^{*} p=0.004\right.$, paired $t$ test, $n=8$ experiments, bars indicate SEM).

through glutamate receptors that occurs following spontaneous synaptic vesicle fusion at resting synapses (Fig. 1B, Movie 1). The fluorescent $\mathrm{Ca}^{2+}$ signal peaked in $\sim 50 \mathrm{~ms}$ and decayed to baseline by $300-500 \mathrm{~ms}$, with a change in baseline fluorescence $(\Delta F)$ of $40-100 \%$ (Fig. $1 B$ ). Subsequent events at the same site produced similar $\mathrm{Ca}^{2+}$ profiles, allowing us to follow vesicle fusion at defined ROIs over continuous imaging sessions ranging from 4 to $10 \mathrm{~min}$, during which spontaneous release frequency and average $\Delta F$ signal was stable (Fig. $1 E, F$ ). We limited our analysis to this time window, as longer-term imaging tended to reduce mini frequency and decrease $\Delta F$. Distinct ROIs defined by spatially segregated $\mathrm{Ca}^{2+}$ signals could be identified over the entire arbor 
(see Figs. $1 C, 3 A$, Movie 1), providing spatial information over time for release at multiple sites. Spontaneous $\mathrm{Ca}^{2+}$ signals detected optically were correlated with simultaneous electrophysiological recordings of minis at the NMJ for $99.8 \%$ of the events (Fig. 2A). Examination of the few $\mathrm{Ca}^{2+}$ imaging events that were not obvious in physiological traces often revealed complex spontaneous voltage events with multiple peaks that were easy to separate optically due to their spatial segregation along the axonal arbor (Fig. 2B). In addition, $82 \%$ of spontaneous events detected by simultaneous physiological recordings were observed optically. We preselected relatively flat NMJs for our analysis and recorded from a single focal plane, but the remaining $18 \%$ of the events were likely from active zones residing outside of the focal plane. Postimaging fixation of preparations allowed staining for the active zone resident protein Bruchpilot (BRP), which provided a rough alignment of ROIs detected by postsynaptic $\mathrm{Ca}^{2+}$ influx in live NMJs with the underlying active zones in fixed tissue (Fig. 1D). We observed that a small population of BRPlabeled active zones was not identified as sites of vesicle fusion in our imaging sessions (Fig. 1D). Although these active zones could represent sites out of the plane of focus, many such sites were active during evoked stimulation of the NMJ (see below).

\section{Characterization of spontaneous release properties at individual active zones}

Having established a preparation to visualize release at individual active zones, we next performed imaging of spontaneous activity at muscle 4 NMJs in eight animals to define the general rules for spontaneous fusion at single release sites. The average duration of each continuous imaging session was $\sim 5 \mathrm{~min}(293 \pm 20 \mathrm{~s})$ with an acquisition rate of $8-11 \mathrm{~Hz}$. We identified $184.5 \pm 7.4$ ROIs per NMJ, with an average event frequency of $2.29 \pm 0.4 \mathrm{~Hz}$. Across the population of ROIs, spontaneous release events occurred at a rate of $0.012 \pm 0.001 \mathrm{~Hz}$ per ROI, indicating a spontaneous vesicle was released on average once every $81 \mathrm{~s}$ per active zone. However, spontaneous release probability per active zone was not uniform across the population. Indeed, the distribution of spontaneous release probability was highly non-Poisson (Fig. $3 B$ ), with far too many low probability release sites, and a smaller subset of highly active release sites. To determine whether there was a spatial logic to release probability along the axonal arbor, we generated heat maps of ROI release rates for each NMJ (Fig. $3 C$ ). We found that high probability release sites could be localized immediately adjacent to low probability sites (Fig. $3 C$ ), indicating spontaneous release probability can be regulated at the level of individual active zones. Unlike what has been previously reported for evoked release (Peled and Isacoff, 2011), terminal boutons of axonal arbors showed no increase in high spontaneous release probability ROIs (Fig. $4 A-C$ ). However, there was an increased correlation of release probability among neighboring sites that extended to $\mathrm{a} \sim 2.5 \mu \mathrm{M}$ area window from the most
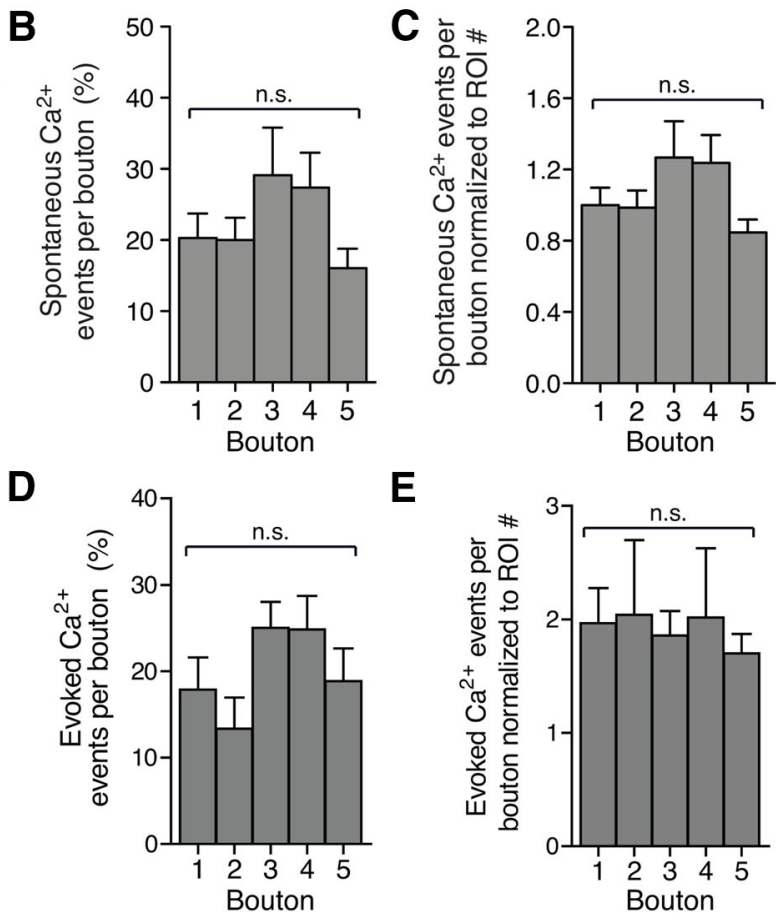

Figure 4. The distribution of spontaneous and evoked fusion rates is uniform across boutons. $\boldsymbol{A}$, Schematic representation of bouton numbering at muscle 4 NMJs that was used to calculate vesicle fusion rates at distal (1) and proximal (2-5) boutons. The ( 位e to the total number of events detected at all 5 boutons. No significant enhancement of release activity at any particular bouton was observed. C, E, Spontaneous and evoked release rates for each bouton were normalized to the number of ROIs detected in each bouton. Again, no significant enhancement of release was detected among boutons.

active ROIs along the arbor (Fig. 3D-F), which is approximately the size of a single synaptic bouton. As such, signaling events that regulate release probability may be compartmentalized within individual boutons and coordinately affect a subpopulation of neighboring active zones. We next examined whether a spontaneous release event occurring at a release site would affect the time course of subsequent release events. We hypothesized there might be a required delay that would potentially arise from vesicle depletion. In contrast, we found that single sites could release a second vesicle within several hundred milliseconds of the first event, and could release at a max rate of 5 vesicles per minute.

\section{Characterization of evoked release properties at individual active zones}

In contrast to spontaneous fusion, which is $\mathrm{Ca}^{2+}$-independent at Drosophila NMJs (Huntwork and Littleton, 2007; Lee et al., 2013), evoked release is triggered following action potential propagation into presynaptic terminals and the influx of $\mathrm{Ca}^{2+}$ at active zones. The synchronous fusion of vesicles across many active zones is predicted to give rise to a postsynaptic current that underlies evoked synaptic transmission. We were able to visualize evoked responses occurring at single ROIs by using a modest extracellular $\mathrm{Ca}^{2+}$ concentration that lowered release probability, thereby reducing the number of vesicles released following an action potential (Movie 1). This allowed a number of critical questions in synaptic function to be addressed using the NMJ preparation. For example, do all active zones participate in both spontaneous and evoked fusion? Is release probability correlated between the two modes of vesicle fusion, and if not, is there a spatial logic to the organization of individual release sites along an axonal arbor? Do single synaptic vesicles fuse at individual 


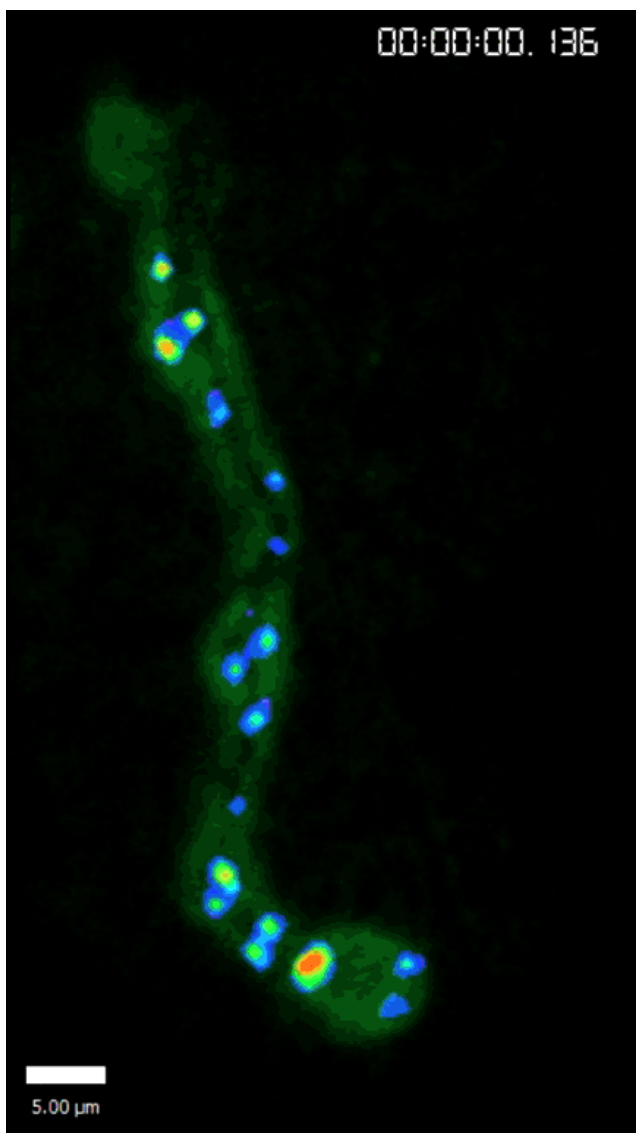

Movie 1. Spontaneous and evoked fusion imaged at muscle 4 NMJs. Background subtracted myrGCaMP5 fluorescence signal is shown for spontaneous $\mathrm{Ca}^{2+}$ activity (first segment) or following evoked action potentials (second segment) during $0.3 \mathrm{~Hz}$ nerve stimulation at muscle 4 NMJs with an acquisition rate of $8 \mathrm{~Hz}$.

active zones during an evoked response, or can multiple vesicles fuse from single active zones? To address these questions, we analyzed spontaneous and evoked release at muscle 4 NMJs in seven animals where the nerve was stimulated at low frequency. Evoked signals were easily distinguished from spontaneous events, as they were time locked to the stimulation (Movie 2). Similar to spontaneous fusion, we observed that evoked release probability was not constant across all active zones, with most sites having a low release probability (Fig. $5 A$ ). A small subset of highly active release sites were found in each preparation, as has been previously noted (Peled and Isacoff, 2011). In contrast to prior observations that indicated high probability release sites were clustered in terminal boutons of the axon (Peled and Isacoff, 2011), we found no correlation between bouton number along the arbor and evoked or spontaneous release rates (Fig. $4 D, E$ ). Although it is unclear what accounts for these differences, myrGCaMP5 is a more robust sensor than previous versions, allowing us to detect most all fusion events unlike prior sensors.

\section{Active zones mediating evoked and spontaneous release can} be spatially distinct

We next analyzed whether individual release sites participated in both evoked and spontaneous synaptic transmission by determining the number of evoked and spontaneous events that occurred per ROI during our imaging sessions. We observed that evoked and spontaneous fusion events could occur

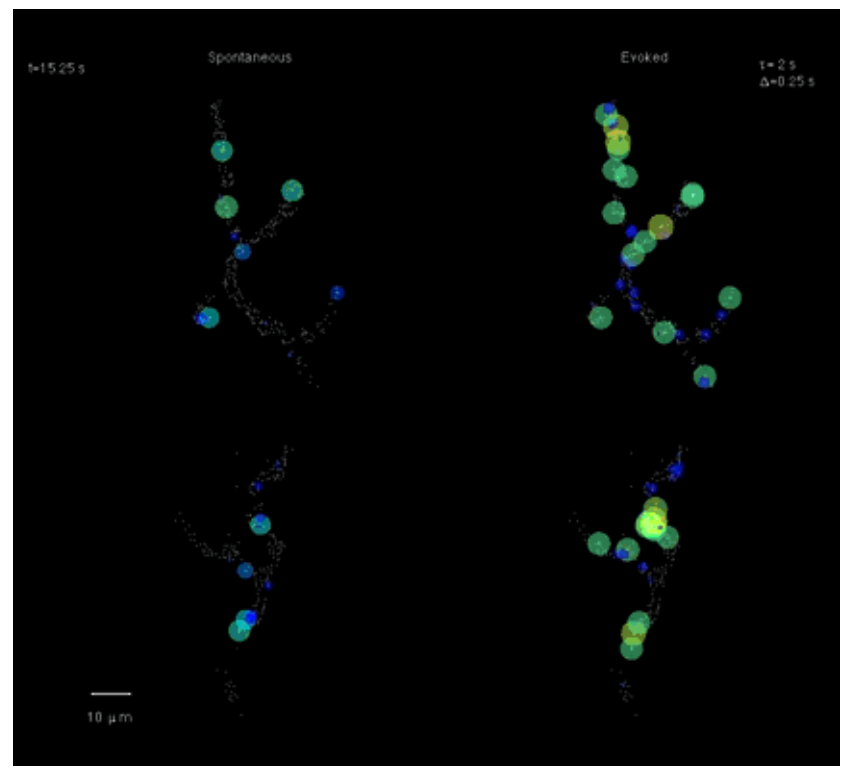

Movie 2. Patterns of evoked and spontaneous activity at muscle 4 NMJs. The pattern of spontaneous (left) and evoked (right) activity recorded concurrently for two separate muscle 4 NMJs (top and bottom) is shown. Spontaneous and evoked events have been separated post hoc for comparison of the temporal dynamics and spatial overlap of the two vesicle release modes. Stimulation events for the two experiments are indicated by white rectangles in the upper left corner of the evoked panels. The location of each ROl identified during the imaging session is indicated by circles. The "activity level" of each ROI is initially set to zero, increases by 1 every time a release event occurs, decays exponentially with a time constant $(\tau=2 \mathrm{~s})$, and is indicated by both the size and color of the ROI (hot and cold colors indicate high and low activity levels, respectively). The relatively slow exponential decay makes it easy to see when ROIs have been coactive within the recent past and overlaps of the exaggerated ROI areas highlight spatial coactivation. The stamp on the upper left shows the time course of events over the acquisition period (binned into $\Delta=0.25$ s intervals).

at the same site, indicating spontaneous and evoked vesicles can fuse from the same population of active zones. Surprisingly, these dual mode active zones accounted for only $41.1 \pm$ $6.0 \%$ of all the ROIs identified in our imaging sessions (Fig. $5 B, C)$. The other two classes of ROIs we observed were evoked only $(36.3 \pm 7.3 \%)$ and spontaneous only sites $(22.3 \pm 4.1 \%)$. Across the seven NMJs examined, the percentage of spontaneous only sites ranged from 10.4 to $38.2 \%$, indicating a significant fraction of release sites are dedicated to spontaneous transmission and do not participate in evoked responses under these recording conditions. One caveat to this conclusion is that some spontaneous fusion events may occur outside of active zones. Several arguments suggest this is not likely to be a major mode of spontaneous release at this synapse. First, if we eliminate all ROIs that contained only a single $\mathrm{Ca}^{2+}$ spike, which could represent fusion occurring outside of an active zone, we still find that $15.2 \pm 4.0 \%$ of all ROIs show only spontaneous events (Fig. $6 B$ ). Second, given the tight clustering of glutamate receptors to active zones at the NMJ (Fig. 1A), we would expect that any fusion event occurring outside of an active zone would have a weaker $\mathrm{Ca}^{2+}$ signal due to transmitters being released further away from glutamate receptor fields that are tethered transynaptically to active zones. However, the $\Delta F$ signals measured for spontaneous events occurring at mixed site ROIs versus spontaneous only ROIs were not different $(\Delta F / F$ for mixed ROIs, $72 \pm 5 \% ; \Delta F / F$ for spontaneous only ROIs, $79 \pm 8 \%$ ). Third, manual inspection of $\mathrm{Ca}^{2+}$ signals at spontaneous only ROIs revealed a similar $\mathrm{Ca}^{2+}$ initiation and diffusion pattern for each event at the ROI, again 
A

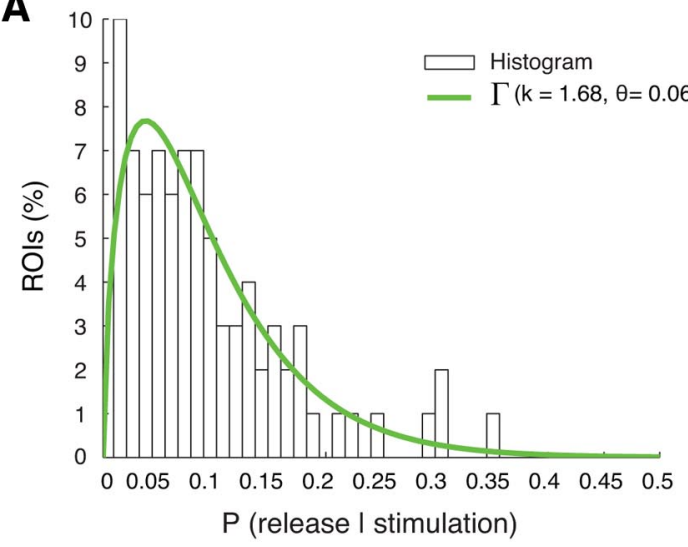

B

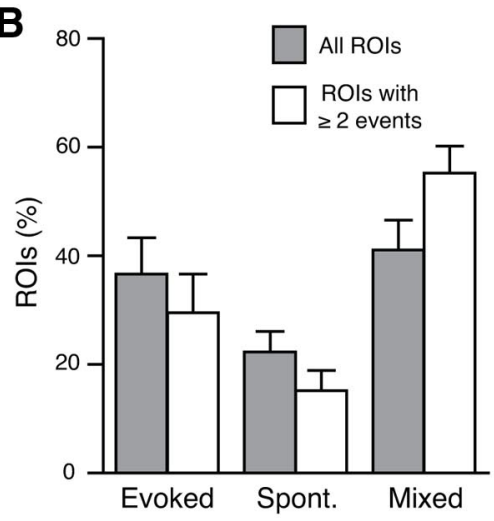

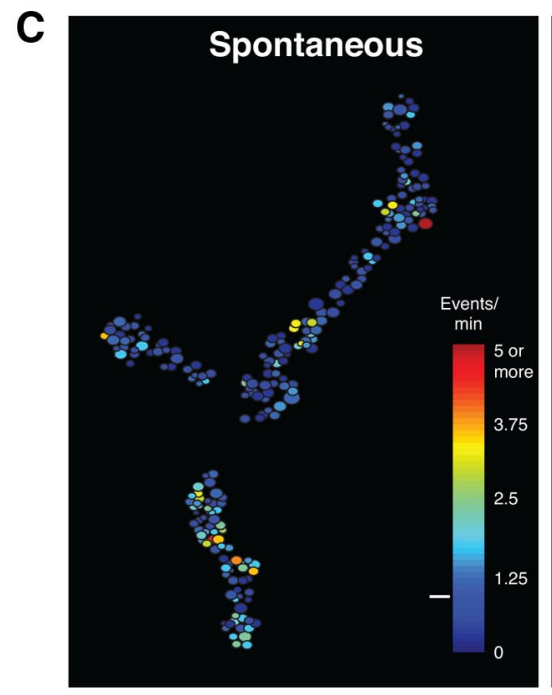
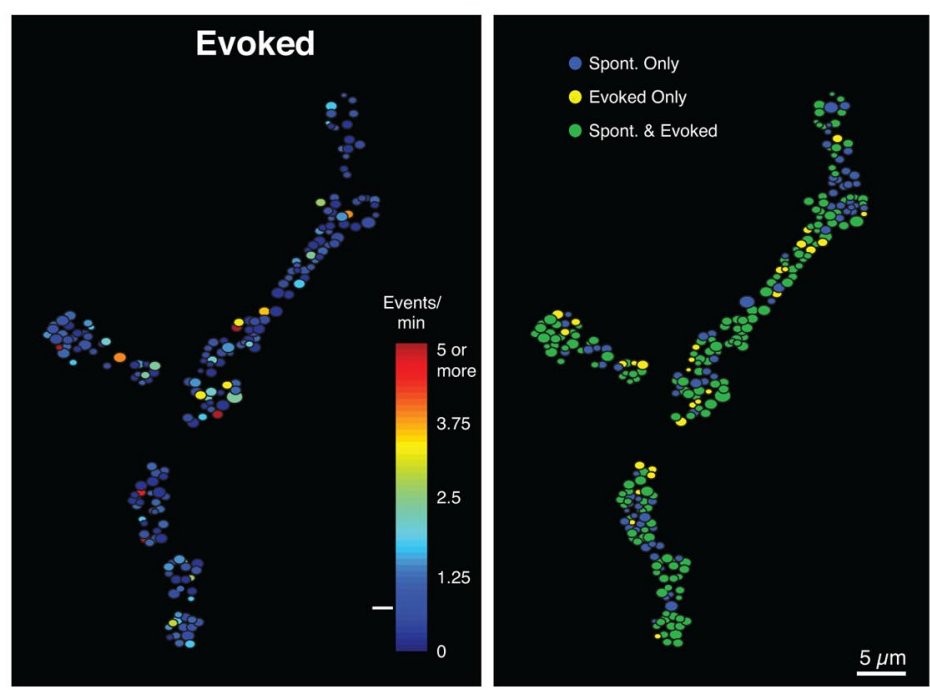

Figure 5. Evoked and spontaneous release events can be segregated across active zones. $A$, The evoked release probability distribution, estimated empirically by binning and counting release probability of all ROls across seven NMJ preparations (bars), is well fit by a gamma distribution (green line, $k=1.68, \theta=0.06, p=0.85, \chi^{2}$ goodness of fit test). $\boldsymbol{B}$, Distribution of vesicle fusion mode [evoked only vs spontaneous only vs evoked and spontaneous (mixed)] over all Rols (gray bars) or ROls with more than two fusion events (white bars) recorded during evoked stimulation at $0.3 \mathrm{~Hz}$ for seven NMJ preparations. C, Heatmaps showing the spatial distribution of synaptic activity for a single NMJ. ROls are coded individually based on spontaneous (left) and evoked (center) event rates. The right panel indicates whether each ROI produced only spontaneous events (blue), only evoked events (yellow), or both (green). Note that release probability is not correlated for the two modes of fusion, as high releasing active zones code preferentially for only one mode of release.

suggesting release was occurring spatially in a similar fashion for each event, consistent with fusion at predetermined docking sites. Finally, the localization of spontaneous only ROIs could often be correlated with the active zone protein BRP (Fig. 6).

Similar to spontaneous only releasing sites, we observed a relatively large population of evoked only ROIs during the short imaging sessions. We wondered whether these sites may represent active zones with a small spontaneous release probability, which coupled with the short imaging windows, could lead to spontaneous events being missed that would occur over a longer imaging session. We therefore analyzed additional $10 \mathrm{~min}$ imaging sessions where we interleaved $2.5 \mathrm{~min}$ periods of spontaneous activity with 2.5 min periods of low frequency nerve stimulation. During this longer paradigm, we found that only $11 \%$ of ROIs showed evoked only responses, indicating a majority of the "evoked-only" sites likely represent ROIs with a very low release probability for spontaneous events, rather than sites that are unable to support spontaneous fusion. In contrast, ROIs with two or more spontaneous fusion events that did not participate in evoked transmission was $23.4 \%$, consistent with shorter imaging sessions.
Properties of active zones participating in both evoked and spontaneous release

We next examined whether multivesicular release at individual active zones could occur during evoked responses (Fig. 7A). A comparison of $\Delta \mathrm{FCa}^{2+}$ signals for spontaneous and evoked release at mixed mode active zones revealed a nearly identical peak response across the population for fusion through either mechanism (Fig. $7 B$ ), suggesting single vesicle fusion at individual active zones is the primary mechanism for evoked release. For $2.1 \%$ of the mixed mode ROIs, we did observe a twofold larger $\Delta F$ for evoked versus spontaneous $\mathrm{Ca}^{2+}$ signals, suggesting a small subset of events may represent fusion of two vesicles at single active zones. Given the presence of spontaneous only active zones detected in our imaging, we next examined whether evoked and spontaneous release probability would be correlated at mixed mode active zones, or if they would be independently regulated. Indeed, we found that spontaneous and evoked release were highly noncorrelated (Figs. 6, 7C), indicating the presence of active zones with a high probability of evoked release and low probability of spontaneous release, and vice versa. These differences in release probability are easily noted in spatial heat maps displaying event rates per ROI for spontaneous and evoked responses at the 


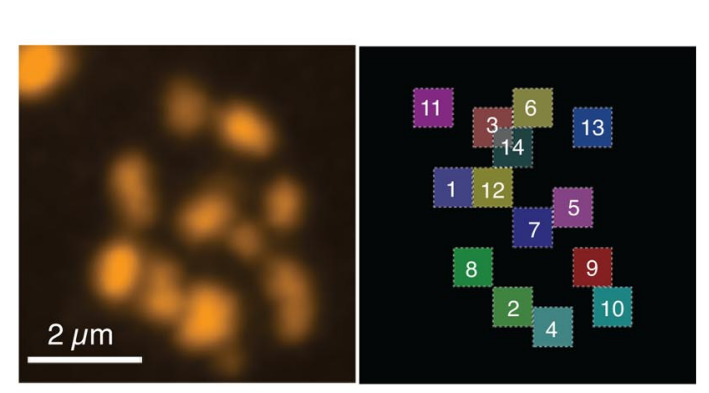

\begin{tabular}{c} 
ROI \\
\hline 1 \\
2 \\
3 \\
4 \\
5 \\
6 \\
7 \\
8 \\
9 \\
10 \\
11 \\
12 \\
13 \\
14
\end{tabular}

$\begin{array}{ll}\text { Event time }(\mathrm{s}) & \text { Evoked events } \\ 3.1 & \text { Spontaneous events }\end{array}$

$6.9,14.5$

217.6, 184.2

$11.6,22.1$

45.8

$172.3,221.6$

$172.8,206.6$

$6.9,172.8$

$0.2,78.5$

$15.6,180.3$

$21.6,27.9,37.4,154.0$

18.3

18.3

214.7
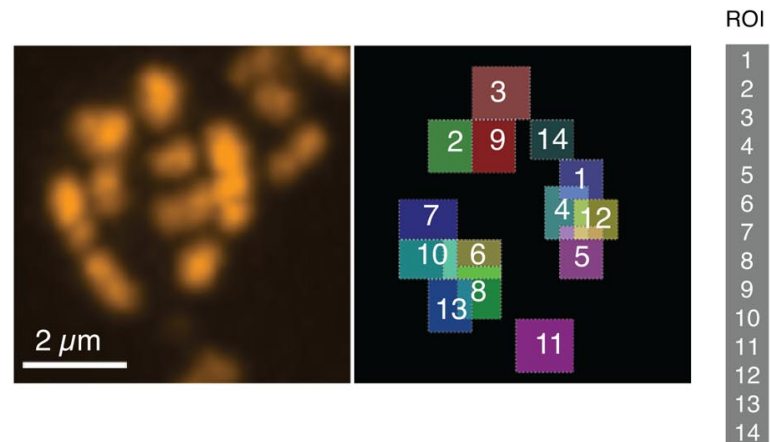

Event time (s)

37.4

$7.1,56.4,116.1$

$3.1,7.6,10.7,22.1,33.6,37.4,48.6,52.4$

$3.1,10.2,14.5,26.0,29.8,30.6,74.9,78.5,90.0,120.2,232.9$

$22.0,202.8,221.6$

$10.9,22.1,29.6,48.6,52.4,74.9,97.6$

$28.4,44.8,86.2,101.4$

202.8

$142.7,165.4,172.8,184.1,191.5,232.9$

$55.4,139.1,176.6,180.4,184.1,210.4$

197.7

217.8

172.8

225.4
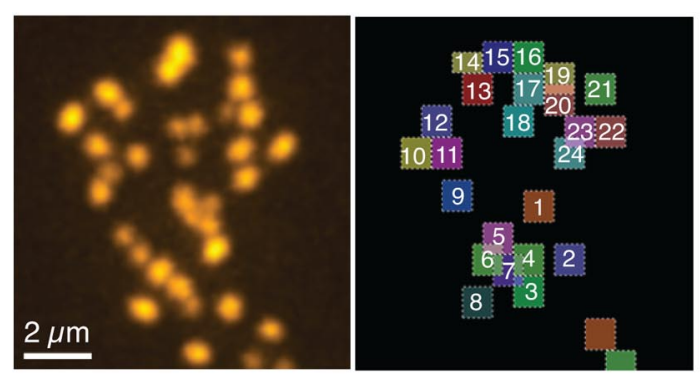

ROI Event time (s)

1
2
3
4
5
6
7
8
9
10
11
12
13
14
15
16
17
18
19
20
21
22
23
24

70.5

$5.5,52.8,120.7$

156.2

$40.9,123.7,182.8$

0.1

$26.2,35.0,61.8,64.4$

165.0

$8.5,17.4,26.2,35.0,37.9,43.9,49.8,58.7,61.7,67.5,76.3$

$85.2,100.0,120.7,126.6,153.2$

$6.9,37.9,40.9,135.5,197.5,199.7,199.9$

$76.3,123.7,153.2$

$43.9,58.7$

40.9

14.3

$11.5,14.4,61.7,82.2$

$32.0,191.7$

98.0

$138.5,157.5$

$12.8,17.7$

121.1

2.6, 58.5

157.1

$14.7,35.0,156.2,159.3$

$14.4,126.6,159.2$

$17.4,165.0$

Figure 6. Single bouton reconstructions of BRP localization and ROl event activity. Three individual boutons are shown with the corresponding BRP labeling postfixation, and the ROIs seen during imaging at $8 \mathrm{~Hz}$ on the right. For each ROl, the timeframe for which a spontaneous (labeled in black) or evoked (labeled in red) event occurred is shown.

same NMJ (Fig. 5C), and indicate that spontaneous and evoked fusion are differentially regulated at individual active zones. Similar to the proximity effects observed for spontaneous fusion, sites with higher release probability for both modes of fusion were clustered near the most highly active ROIs within a $2.5 \mu \mathrm{m}$ radius (Fig. 7D).

\section{Discussion}

Using an optical tool to measure postsynaptic responses to neurotransmitter release from individual active zones, we characterized the rules for spontaneous and evoked fusion, as well as their spatial and temporal relationship. Release probability is not correlated at individual active zones for the two pathways, suggesting they are independently regulated. How could spontaneous and evoked release be differentially regulated at the same active zone? One possibility is that distinct vesicle pools give rise to each mode of release with limited cross talk (Fredj and Burrone, 2009; Chung et al., 2010; Sara et al., 2011), although no molecular marker has yet been identified that can differentiate the two vesicle pools at Drosophila synapses. Another possibility is that differences in the presynaptic vesicle fusion machinery regulate spontaneous and evoked release (Ramirez and Kavalali, 2011). Spontaneously fusing vesicles could differ in their content of SNARE-binding regulatory proteins like the $\mathrm{Ca}^{2+}$ sensor synaptotagmin or the fusion clamp complexin. Regulation of complexin levels per active zone would be an enticing mechanism to control spontaneous release probability, as genetic manipulation of its levels bidirectionally controls mini event fre- 
A Model 1

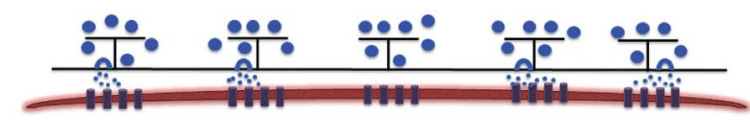

Model 2

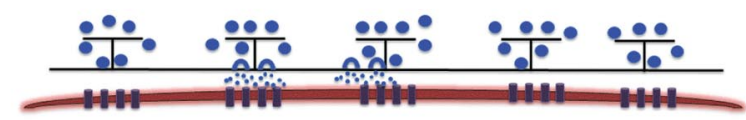

C

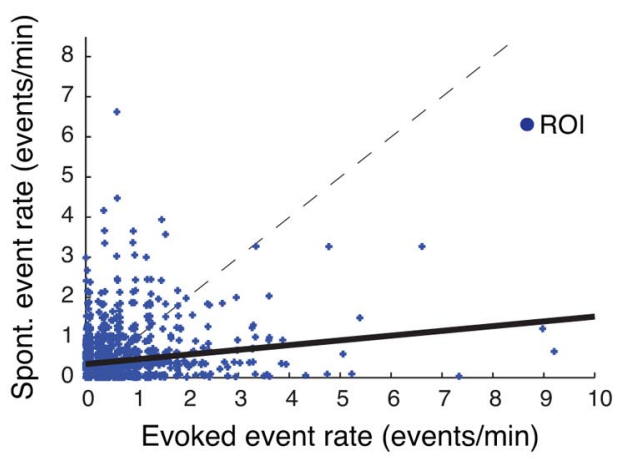

B

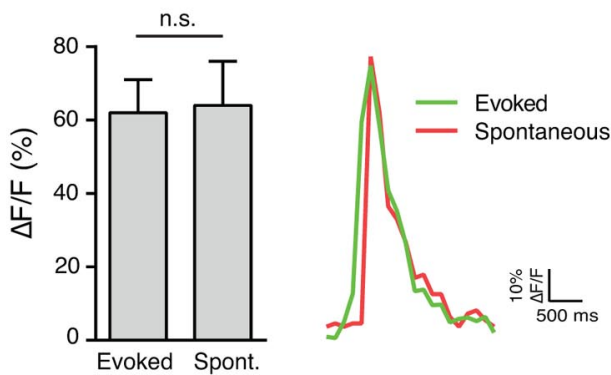

D

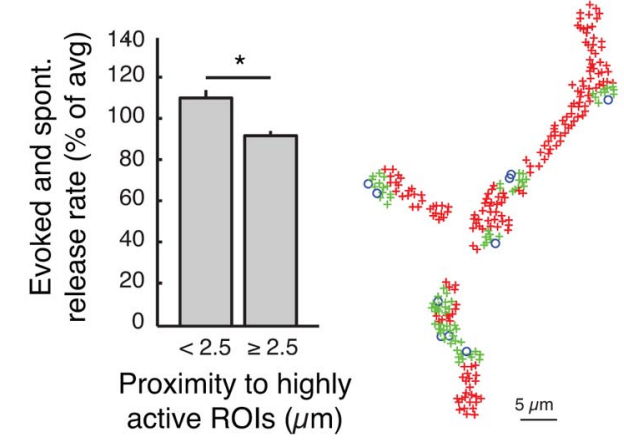

Figure 7. Comparison of spontaneous and evoked release properties at active zones. $A$, Model depicting two modes of evoked release is shown. In Model 1 , action potentials trigger single vesicle fusion events across a subset of individual active zones. In Model 2 , multiple vesicles can release from single active zones. $\Delta F$ measurements for spontaneous or evoked events at mixed ROls reveal identical $\Delta F$ signals across the entire population, suggesting multivesicular release from a single active zone is not commonly observed at the NMJ. $\boldsymbol{B}$, Representative $\Delta F$ traces for spontaneous and evoked release events are shown on the right. C, Comparison of the spontaneous event rate for a single ROI as a function of its evoked event rate for every data point across seven NMJs. Evoked and spontaneous release rate per $R 01$ are poorly correlated $(\rho=0.161, p<0.001)$. The linear regression line has a very low slope $(0.118$, solid line $)$ and is a poor fit of the data $\left(R^{2}=0.03\right.$, dashed line depicts hypothetical 1:1 correlation). $\boldsymbol{D}$, Regions near highly active ROIs have a significantly higher event rate (combined spontaneous and evoked, population map shown at right) than those farther away $\left({ }^{*} p=0.021\right.$, paired $t$ test, $n=7$ experiments, bars indicate SEM).

quency (Jorquera et al., 2012). Likewise, modulation of complexin function through phosphorylation would be an attractive mechanism to independently regulate the rate of spontaneous release, which is enhanced following highfrequency stimulation at Drosophila NMJs (Yoshihara et al., 2005; Ataman et al., 2008; Barber et al., 2009). Spontaneously fusing synaptic vesicles could also be regulated by modification of the SNARE proteins. For example, the $\mathrm{H}_{\mathrm{abc}}$-domain of syntaxin-1 was recently found to be required for spontaneous, but not evoked, release (Zhou et al., 2013).

Our results show that $22 \%$ of all active zones only participate in spontaneous fusion, suggesting the existence of an independent information channel where a population of postsynaptic glutamate receptors is activated only in response to spontaneous neurotransmission. It will be of interest to determine whether these active zones are plastic in their release properties, or whether they are permanently transmitting spontaneous only information. In terms of molecular mechanisms, spontaneous only sites may lack voltage-gated $\mathrm{Ca}^{2+}$ channels, have voltage gated $\mathrm{Ca}^{2+}$ channels that have been silenced through posttranslational modifications like phosphorylation, or lack the fusion machinery required for evoked neurotransmission.

Unlike evoked neurotransmission, which has essential roles in triggering action potentials in postsynaptic partners, the role of subthreshold spontaneous fusion events is less clear. Prior studies have linked enhancements in spontaneous fusion to activitydependent growth of the Drosophila NMJ (Huntwork and Littleton, 2007; Ataman et al., 2008), suggesting a possible role for spontaneous only active zones in the regulation of structural plas- ticity. Spontaneous release has also been implicated in many other forms of synaptic modulation and plasticity (Sutton et al., 2006; Lee et al., 2010; Kavalali et al., 2011; Gonzalez-Islas et al., 2012; Jin et al., 2012; Nosyreva et al., 2013). Interestingly, spontaneous release at hippocampal synapses has been found to activate a distinct set of signaling cascades compared with evoked release (Sutton et al., 2004, 2007). These results suggest spontaneous only active zones could have a wide influence over neuronal signaling. The rate of spontaneous release we observed of one vesicle fusion event every $81 \mathrm{~s}$ is very similar to the rate of spontaneous fusion observed at hippocampal synapses (one event every $91 \mathrm{~s}$ ) (Murthy and Stevens, 1999). It also corresponds approximately to the time course for homeostatic plasticity that has been observed at the Drosophila NMJ, where blockage of postsynaptic glutamate receptors rapidly induces an increased release probability within minutes (Frank et al., 2006). It will be interesting to determine whether individual postsynaptic sites have a "molecular clock" for $\mathrm{Ca}^{2+}$ signals that can detect a change in spontaneous release and initiate a retrograde signal if fusion is not detected over a specified time window. Using the current toolkit to generate individual heat maps for release probability for the two modes of fusion (Fig. $5 \mathrm{C}$ ), one can now examine how individual active zone release properties are changed following synaptic potentiation or in various genetic backgrounds disrupting active zone or synaptic vesicle proteins. Whether release properties are regulated by individual active zone components or local synaptic vesicle pools tethered to release sites will be an important question for future studies. 


\section{Notes}

Supplemental material for this article is available at web.mit.edu/flybrain/ littletonlab/Melom, additional Movies of GCaMP5 imaging at Drosophila synapses. This material has not been peer reviewed.

\section{References}

Ataman B, Ashley J, Gorczyca M, Ramachandran P, Fouquet W, Sigrist SJ, Budnik V (2008) Rapid activity-dependent modifications in synaptic structure and function require bidirectional Wnt signaling. Neuron 57: 705-718. CrossRef Medline

Atasoy D, Ertunc M, Moulder KL, Blackwell J, Chung C, Su J, Kavalali ET (2008) Spontaneous and evoked glutamate release activates two populations of NMDA receptors with limited overlap. J Neurosci 28:1015110166. CrossRef Medline

Barber CF, Jorquera RA, Melom JE, Littleton JT (2009) Postsynaptic regulation of synaptic plasticity by synaptotagmin 4 requires both C2 domains. J Cell Biol 187:295-310. CrossRef Medline

Chung C, Barylko B, Leitz J, Liu X, Kavalali ET (2010) Acute dynamin inhibition dissects synaptic vesicle recycling pathways that drive spontaneous and evoked neurotransmission. J Neurosci 30:1363-1376. CrossRef Medline

Desai SA, Lnenicka GA (2011) Characterization of postsynaptic Ca2 + signals at the Drosophila larval NMJ. J Neurophysiol 106:710-721. CrossRef Medline

Fatt P, Katz B (1952) Spontaneous subthreshold activity at motor nerve endings. J Physiol 117:109-128. Medline

Frank CA, Kennedy MJ, Goold CP, Marek KW, Davis GW (2006) Mechanisms underlying the rapid induction and sustained expression of synaptic homeostasis. Neuron 52:663-677. CrossRef Medline

Fredj NB, Burrone J (2009) A resting pool of vesicles is responsible for spontaneous vesicle fusion at the synapse. Nat Neurosci 12:751-758. CrossRef Medline

Gonzalez-Islas C, Garcia-Bereguiain MA, Wenner P (2012) Tonic and transient endocannabinoid regulation of AMPAergic miniature postsynaptic currents and homeostatic plasticity in embryonic motor networks. J Neurosci 32:13597-13607. CrossRef Medline

Groemer TW, Klingauf J (2007) Synaptic vesicles recycling spontaneously and during activity belong to the same vesicle pool. Nat Neurosci 10:145147. CrossRef Medline

Hua Z, Leal-Ortiz S, Foss SM, Waites CL, Garner CC, Voglmaier SM, Edwards RH (2011) v-SNARE composition distinguishes synaptic vesicle pools. Neuron 71:474-487. CrossRef Medline

Huntwork S, Littleton JT (2007) A complexin fusion clamp regulates spontaneous neurotransmitter release and synaptic growth. Nat Neurosci 10: 1235-1237. CrossRef Medline

Jin I, Puthanveettil S, Udo H, Karl K, Kandel ER, Hawkins RD (2012) Spontaneous transmitter release is critical for the induction of long-term and intermediate-term facilitation in Aplysia. Proc Natl Acad Sci U S A 109: 9131-9136. CrossRef Medline

Jorquera RA, Huntwork-Rodriguez S, Akbergenova Y, Cho RW, Littleton JT (2012) Complexin controls spontaneous and evoked neurotransmitter release by regulating the timing and properties of synaptotagmin activity. J Neurosci 32:18234-18245. CrossRef Medline

Katz B, Miledi R (1969) Spontaneous and evoked activity of motor nerve endings in calcium Ringer. J Physiol 203:689-706. Medline

Kavalali ET, Chung C, Khvotchev M, Leitz J, Nosyreva E, Raingo J, Ramirez DM (2011) Spontaneous neurotransmission: an independent pathway for neuronal signaling? Physiology 26:45-53. CrossRef Medline

Lee J, Guan Z, Akbergenova Y, Littleton JT (2013) Genetic analysis of syn- aptotagmin C2 domain specificity in regulating spontaneous and evoked neurotransmitter release. J Neurosci 33:187-200. CrossRef Medline

Lee MC, Yasuda R, Ehlers MD (2010) Metaplasticity at single glutamatergic synapses. Neuron 66:859-870. CrossRef Medline

Littleton JT, Stern M, Schulze K, Perin M, Bellen HJ (1993) Mutational analysis of Drosophila synaptotagmin demonstrates its essential role in $\mathrm{Ca}(2+)$-activated neurotransmitter release. Cell 74:1125-1134. CrossRef Medline

Maximov A, Shin OH, Liu X, Südhof TC (2007) Synaptotagmin-12, a synaptic vesicle phosphoprotein that modulates spontaneous neurotransmitter release. J Cell Biol 176:113-124. CrossRef Medline

Murthy VN, Stevens CF (1999) Reversal of synaptic vesicle docking at central synapses. Nat Neurosci 2:503-507. CrossRef Medline

Nosyreva E, Szabla K, Autry AE, Ryazanov AG, Monteggia LM, Kavalali ET (2013) Acute suppression of spontaneous neurotransmission drives synaptic potentiation. J Neurosci 33:6990-7002. CrossRef Medline

Pang ZP, Bacaj T, Yang X, Zhou P, Xu W, Südhof TC (2011) Doc2 supports spontaneous synaptic transmission by a $\mathrm{Ca}(2+)$-independent mechanism. Neuron 70:244-251. CrossRef Medline

Peled ES, Isacoff EY (2011) Optical quantal analysis of synaptic transmission in wild-type and rab3-mutant Drosophila motor axons. Nat Neurosci 14:519-526. CrossRef Medline

Ramirez DM, Kavalali ET (2011) Differential regulation of spontaneous and evoked neurotransmitter release at central synapses. Current opinion in neurobiology 21:275-282. CrossRef Medline

Ramirez DM, Khvotchev M, Trauterman B, Kavalali ET (2012) Vtila identifies a vesicle pool that preferentially recycles at rest and maintains spontaneous neurotransmission. Neuron 73:121-134. CrossRef Medline

Sara Y, Virmani T, Deák F, Liu X, Kavalali ET (2005) An isolated pool of vesicles recycles at rest and drives spontaneous neurotransmission. Neuron 45:563-573. CrossRef Medline

Sara Y, Bal M, Adachi M, Monteggia LM, Kavalali ET (2011) Use-dependent AMPA receptor block reveals segregation of spontaneous and evoked glutamatergic neurotransmission. J Neurosci 31:5378-5382. CrossRef Medline

Söllner T, Whiteheart SW, Brunner M, Erdjument-Bromage H, Geromanos S, Tempst P, Rothman JE (1993) SNAP receptors implicated in vesicle targeting and fusion. Nature 362:318-324. CrossRef Medline

Sutton MA, Wall NR, Aakalu GN, Schuman EM (2004) Regulation of dendritic protein synthesis by miniature synaptic events. Science 304:19791983. CrossRef Medline

Sutton MA, Ito HT, Cressy P, Kempf C, Woo JC, Schuman EM (2006) Miniature neurotransmission stabilizes synaptic function via tonic suppression of local dendritic protein synthesis. Cell 125:785-799. CrossRef Medline

Sutton MA, Taylor AM, Ito HT, Pham A, Schuman EM (2007) Postsynaptic decoding of neural activity: eEF2 as a biochemical sensor coupling miniature synaptic transmission to local protein synthesis. Neuron 55:648 661. CrossRef Medline

Yoshihara M, Ueda A, Zhang D, Deitcher DL, Schwarz TL, Kidokoro Y (1999) Selective effects of neuronal-synaptobrevin mutations on transmitter release evoked by sustained versus transient $\mathrm{Ca} 2+$ increases and by cAMP. J Neurosci 19:2432-2441. Medline

Yoshihara M, Adolfsen B, Galle KT, Littleton JT (2005) Retrograde signaling by Syt 4 induces presynaptic release and synapse-specific growth. Science 310:858-863. CrossRef Medline

Zhou P, Pang ZP, Yang X, Zhang Y, Rosenmund C, Bacaj T, Südhof TC (2013) Syntaxin-1 N-peptide and Habc-domain perform distinct essential functions in synaptic vesicle fusion. EMBO J 32:159-171. Medline 